\title{
CYP2D6 genetic polymorphism and psychiatry patients' hospitalization period
}

A letter in response to: Ruaño G, Szarek BL, Villagra D et al. Length of psychiatric hospitalization is correlated with CYP2D6 functional status in inpatients with major depressive disorder. Biomarkers Med. 7(3), 429-439 (2013).

The article by Ruaño et al. analyzes whether CYP2D6 has an effect on length of hospital stay (LOS) in inpatients with major depressive disorder [1]. The aim is of interest because cost-effectiveness studies must be performed for the clinical implementation of pharmacogenetics $[2,3]$. The reported findings showed longer hospital stays in individuals carrying CYP2D 6 alleles associated with deficient enzyme function (metabolic reserve <2) compared with metabolizers with normal or increased function (metabolic reserve $\geq 2 ; 7.8$ vs 5.7 days, respectively; $p=0.002$ ). The interpretation of these results is discussed below.

\section{Point 1}

A quantitative 'drug metabolic reserve index' was calculated based on each individual's CYP2DG genotype. In other words, a numeric value was assigned to CYP2D6 alleles according to a theoretical' functional value: 0 to $C Y P 2 D 6$ alleles $* 4$, ${ }^{*} 4 \mathrm{XN},{ }^{*} 5,{ }^{*} 6,{ }^{*} 7,{ }^{*} 8,{ }^{*} 11,{ }^{*} 12,{ }^{*} 14$ and ${ }^{*} 15 ; 0.5$ to alleles ${ }^{*}$, ${ }^{*} 10,{ }^{*} 17$ and ${ }^{*} 41 ; 1$ to alleles ${ }^{*} 1$ and ${ }^{*} 2$; 1.5 and ${ }^{*} 2 a$; and 2 to the duplication/multiplication of alleles ${ }^{*} 1$ and ${ }^{*} 2$. Scores on the drug metabolic reserve index ranged from 0 to 3 in the population, and individuals were categorized into those with a score $<2(\mathrm{n}=66)$ and individuals with a score $\geq 2(n=83)$. The former category clustered all individuals with genotypes related to null or reduced enzyme activity, whereas the latter category comprised those with genotypes signaling normal or increased CYP2D6 enzyme activity. However, the frequency of poor metabolizers or ultrarapid metabolizers is not clearly stated, and whether this population is different from others in the literature needs to be clarified. It seems that it is biased, showing a higher percentage of individuals carrying alleles related to deficient activity. The study of a population of psychiatric patients under pharmacological treatment might be linked to under- or over-representation of phenotypes potentially related to adverse drug reactions, lack of efficacy or compliance [4,5]. In addition, the functional classification of CYP2D6 activity from genotype should be discussed, since it is based on a theoretical enzyme activity value that is in turn the result of a theoretical value given to each allele. Therefore, this classification may be biased by the existence of some overlap between these theoretically defined groups. The only phenotype group that it is accurately defined by genotype is that of poor metabolizers carrying two alleles with a value of 0 . However, there may be people without enzyme activity carrying copies of active genes as a result of CYP2D6 inhibition during treatment [6]. In addition, other defined CYP2D6 metabolism indices ('metabolic alteration' and 'allele alteration') were calculated, but were not discussed in relation to length of hospitalization.

\section{Point 2}

On the other hand, the authors partially acknowledged observational design as a main limitation of the study, including patients exposed to a variety of treatments. In spite of this type of design, several factors that were not controlled could have been analyzed. In keeping with that fact, it is reported that almost all patients were treated with antidepressants during hospitalization (98\%), but that approximately $63 \%$ also received antipsychotics, and a smaller proportion anticonvulsants. Despite the fact that they stated that lack of control of the number of medications, individuals could have been classified into those under polypharmacy and those not. It appears that approximately $57 \%$ of the patients could have been under polypharmacy of antidepressants and/or antipsychotics and/or anticonvulsants during the hospitalization period. Because polypharmacy may increase the probability of adverse events, which are related to longer hospital stays, this factor should be analyzed in future studies [7]. Furthermore, future studies addressing this question should establish whether or not there are differences between patients taking

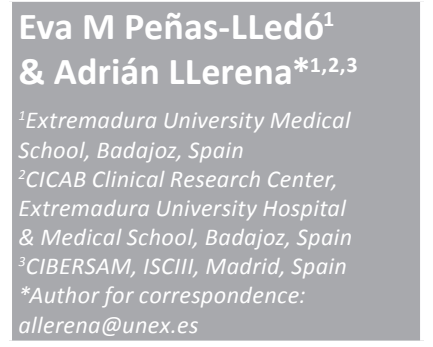

Future
Medicine 
CYP2D6 substrates and those that are under treatment with other substrates, as well as the number of CYP2D6 substrates and the relevance of this metabolic path for each drug. These last aspects were calculated for the present study but were not analyzed later. This is a major point for discussion, since it is the main hypothesis of the study. LOS should be related to the CYP2D 6 genotype only in those taking CYP2D6 substrates. In addition, LOS should be related to genotype controlling for number of CYP2D6 substrates and drug dosage $[7,8]$, since in a naturalistic study dosages should have already been empirically adjusted to the actual CYP2D6 hydroxylation capacity. Moreover, the relationship between genotypes and drug dosage could help to understand the nature of the association found with $\operatorname{LOS}[3,4]$.

\section{Point 3}

Besides the implication of CYP2D6 in drug metabolism, the potential relationship with psychopathology must be discussed $[9,10]$. It is highlighted that almost all patients (93\%) were suicidal, and most had received psychotropic treatment for major depressive disorder prior to admission. Since severity of suicide, as well as suicidal attempt [11,12] and completion [13], have all been related to increased CYP2D6 enzyme activity regardless of drug treatment, this factor could have also affected present findings. Furthermore, was the history of drug treatment (lack of efficacy or intoxication prior to admission) taken into account to understand LOS? It might be expected that poor metabolizers were hospitalized owing to drug intoxication and needed a longer stay in hospital to get back to normal.

\section{Point 4}

Other important demographic and clinical factors, such as ethnic background and comorbid psychiatric diagnoses, were very heterogenous in the population and could also influence findings [10]. Consistently, LOS might be better explained by ethnicity $[3,14]$.

While all these factors should be controlled in future naturalistic studies, the present study is of enormous value for the development of pharmacogenetics in clinical practice.

\section{Disclaimer}

This work is the opinion of the authors and does not represent the views of Future Medicine or its employees.

\section{Financial \& competing interests disclosure}

The authors' research in this area is supported by grants from the Spanish Instituto de Salud Carlos III, European UnionFEDER PI10/02758, and CIBERSAM, Gobierno de Extremadura, Consejería de Empleo, Empresa e Innovación and European Union-FEDER Fondo Social Europeo grant no. BS10023. The authors have no other relevant affliations or financial involvement with any organization or entity with a financial interest in or financial conflict with the subject matter or materials discussed in the manuscript apart from those disclosed.

No writing assistance was utilized in the production of this manuscript.

\section{References}

1 Ruaño G, Szarek BL, Villagra D et al. Length of psychiatric hospitalization is correlated with CYP2D6 functional status in inpatients with major depressive disorder. Biomarkers Med. 7(3), 429-439 (2013).

2 Rodríguez-Antona C, Gurwitz D, de Leon J et al. CYP2D6 genotyping for psychiatric patients treated with risperidone: considerations for cost-effectiveness studies. Pharmacogenomics 10(4), 685-699 (2009).

3 LLerena A, Dorado P, Peñas-Lledó EM. Pharmacogenetics of debrisoquine and its use as a marker for CYP2D6 hydroxylation capacity. Pharmacogenomics 10(1), 17-28 (2009).

4 LLerena A, Dorado P, Peñas-Lledó EM et al. Low frequency of CYP2D6 poor metabolizers among schizophrenia patients.

Pharmacogenomics J. 7(6), 408-410 (2007).

5 Peñas-Lledó EM, Trejo HD, Dorado P et al. CYP2D6 ultrarapid metabolism and early dropout from fluoxetine or amitriptyline monotherapy treatment in major depressive patients. Mol. Psychiatry 18(1), 8-9 (2013).

6 González I, Peñas-Lledó EM, Pérez B et al. Relation between CYP2D6 phenotype and genotype and personality in healthy volunteers. Pharmacogenomics 9(7), 833-840 (2008).

7 Berecz R, Dorado P, de la Rubia A et al. The role of cytochrome $\mathrm{P} 450$ enzymes in the metabolism of risperidone and its clinical relevance for drug interactions. Current Drug Targets 5(6), 573-579 (2004).

8 LLerena A, Berecz R, de la Rubia A et al. Effect of thioridazine dosage on the debrisoquine hydroxylation phenotype in psychiatric patients with different CYP2D6genotypes. Ther. Drug Monitor. 23(6), 616-620 (2001).

9 Peñas-LLedó EM, Dorado P, Pacheco R et al. Relation between CYP2D6 genotype, personality, neurocognition and overall psychopathology in healthy volunteers. Pharmacogenomics 10(7), 1111-1120 (2009).

10 Blasco-Fontecilla H, Peñas-Lledó E, VaqueroLorenzo C et al. CYP2D6 polymorphism and mental and personality disorders in suicide attempters. J. Pers. Disord. doi:10.1521/ pedi_2013_27_080 (2013) (Epub ahead of print).

11 Peñas-Lledó EM, Blasco-Fontecilla H, Dorado P et al. CYP2D6 and the severity of suicide attempts. Pharmacogenomics 13(2), 179-184 (2012).

12 Peñas-Lledó EM, Dorado P, Agüera Z et al. High risk of lifetime history of suicide attempts among CYP2D6 ultrarapid metabolizers with eating disorders. Mol. Psychiatry 16(7), 691-692 (2011).

13 Zackrisson AL, Lindblom B, Ahlner J. High frequency of occurrence of CYP2D 6 gene duplication/multiduplication indicating ultrarapid metabolism among suicide cases. Clin. Pharmacol. Ther. 88(3), 354-359 (2010).

14 LLerena A, Dorado P, Ramírez R et al. CYP2D6 genotype and debrisoquine hydroxylation phenotype in Cubans and Nicaraguans. Pharmacogenomics J. 12(2), 176-183 (2012). 